\title{
Rapid Classification and Treatment Algorithm of Cardiogenic Shock Complicating Acute Coronary Syndromes: The SAVE ACS Classification
}

\author{
Vasileios Panoulas ${ }^{10}{ }^{1,2}$ and Charles Ilsley ${ }^{1}$ \\ ${ }^{1}$ Department of Cardiology, Royal Brompton and Harefield Hospitals, Guy's and St Thomas' NHS Foundation Trust, \\ Harefield, UK \\ ${ }^{2}$ Cardiovascular Sciences, National Heart and Lung Institute, Imperial College London, London, UK
}

Correspondence should be addressed to Vasileios Panoulas; v.panoulas@imperial.ac.uk

Received 25 March 2021; Accepted 20 December 2021; Published 12 January 2022

Academic Editor: Shenghua Zhou

Copyright (C) 2022 Vasileios Panoulas and Charles Ilsley. This is an open access article distributed under the Creative Commons Attribution License, which permits unrestricted use, distribution, and reproduction in any medium, provided the original work is properly cited.

\begin{abstract}
Introduction. We aimed to identify the independent "frontline" predictors of 30-day mortality in patients with acute coronary syndromes (ACS) and propose a rapid cardiogenic shock (CS) classification and management pathway. Materials and Methods. From 2011 to 2019, a total of 11439 incident ACS patients were treated in our institution. Forward conditional logistic regression analysis was performed to determine the "frontline" predictors of 30 day mortality. The $C$-statistic assessed the discriminatory power of the model. As a validation cohort, we used 431 incident ACS patients admitted from January 1, 2020, to July 20, 2020. Results. Independent predictors of 30 -day mortality included age (OR 1.05; 95\% CI 1.04 to $1.07, p<0.001$ ), intubation (OR 7.4; $95 \%$ CI 4.3 to $12.74, p<0.001$ ), LV systolic impairment ( $\mathrm{OR}_{\text {severe vs normal }} 1.98 ; 95 \% \mathrm{CI} 1.14$ to $3.42, p=0.015$, OR moderate vs normal $1.84 ; 95 \% \mathrm{CI}$ 1.09 to $3.1, p=0.022$ ), serum lactate (OR $1.25 ; 95 \%$ CI 1.12 to $1.41, p<0.001$ ), base excess (OR $1.1 ; 95 \%$ CI 1.04 to $1.07, p<0.001$ ), and systolic blood pressure (OR 0.99; $95 \%$ CI 0.982 to $0.999, p=0.024$ ). The model discrimination was excellent with an area under the curve (AUC) of 0.879 (0.851 to 0.908) ( $p<0.001$ ). Based on these predictors, we created the SAVE (SBP, Arterial blood gas, and left Ventricular Ejection fraction) ACS classification, which showed good discrimination for 30-day AUC 0.814 (0.782 to 0.845$)$ and long-term mortality $\left(p_{\text {log-rank }}<0.001\right.$ ). A similar AUC was demonstrated in the validation cohort (AUC 0.815). Conclusions. In the current study, we introduce a rapid way of classifying CS using frontline parameters. The SAVE ACS classification could allow for future randomized studies to explore the benefit of mechanical circulatory support in different CS stages in ACS patients.
\end{abstract}

\section{Introduction}

Cardiogenic shock (CS) is often encountered by frontline cardiac catheterization teams, which are part of primary percutaneous coronary intervention (PPCI) pathways [1]. Even though the aetiology of CS can vary, left ventricular (LV) failure following acute myocardial infarction (MI) remains the most frequent cause accounting for over $40 \%$ of cases [2]. Mechanical complications after MI (ventricular septal rupture, free wall rupture, and acute severe mitral regurgitation) in late presenters or patients that have not been revascularized represent a small proportion of CS cases yet carry considerable morbidity and mortality. [3] CS can also be attributed to non-MI causes, such as decompensated chronic heart failure (often dilated cardiomyopathy), valvular heart disease, myocarditis, stress-induced cardiomyopathy (Takotsubo syndrome), or arrhythmias (atrial or ventricular) [4].

Despite the significant advances in reperfusion therapy and percutaneous mechanical circulatory support (MCS) devices, mortality among patients presenting with CS remains very high, ranging from $25 \%$ to $50 \%[5,6]$. In a recent large cohort of 21,210 patients in London with ST segment elevation myocardial infarction (STEMI), CS was observed in $8.9 \%$ of patients with the incidence increasing over time and high mortality of $45 \%-70 \%$ [1]. It should, however, be 
mentioned that the definition of shock in these databases remains dubious due to the lack of detailed echocardiographic, hemodynamic, and biochemical parameters.

Traditional definitions of cardiogenic shock in major cardiac societies [4] and randomized studies [2] have included a systolic blood pressure of $<90 \mathrm{mmHg}$ (or requiring inotropes to keep SBP $\geq 90 \mathrm{mmHg}$ ) for $30 \mathrm{~min}$ or more and evidence of impaired organ perfusion (on clinical examination, lactate $>2.0 \mathrm{mmol} / \mathrm{L}$ and urine output $<30 \mathrm{mls} / \mathrm{h}$ ). Recently, Baran et al. [7] published an easy-to-remember classification of cardiogenic shock based on clinical, observational, biochemical, and hemodynamic parameters. Even though it is a truly thorough and conceptually easy classification, its extensive definitions, including hemodynamic parameters, render its prompt applicability in the frontline settings difficult. Furthermore, the lack of bedside echocardiographic evaluation is surprising given that often LV systolic impairment precedes SBP drop in acute MI settings and is an independent predictor of outcome in CS patients [8].

Developing an immediate response algorithm (within 5 min), which includes early MCS, is important given that registry evidence suggests improved survival when left ventricular support is initiated early on [9].

In the current study, we aimed to identify the independent "frontline" predictors of 30-day mortality in a large cohort of patients presenting with ACS and create a straightforward ACS cardiogenic shock classification that could assist physicians in decision making for early MCS deployment [2].

\section{Methods}

From January 2011 to December 2019, a total of 17908 PCIs were performed in Harefield Hospital (Royal Brompton and Harefield NHS Foundation Trust, London, UK). Of those, 12458 were in the context of ACS, whereas 11,439 were incident presentations within this time frame, excluding those ones with mechanical complications. As a validation cohort, we used 431 incident ACS patients undergoing PCI in our institution from January 1, 2020, to July 20, 2020.

Routine clinical data on demographics, clinical characteristics on presentation, admission observations, electrocardiogram, bedside LV function (prior to procedure), arterial blood gas data (on admission, prior to procedure), past medical history, and procedural characteristics were prospectively collected. Cardiac arrest was classified as follows: EMD (electromechanical dissociation), asystole, VT (ventricular tachycardia), VF (ventricular fibrillation), or unknown.

All patients from the derivation cohort were followed up from the time of the procedure until death or censored 01.06.2020. The primary outcome was 30-day mortality. Vital status was ascertained using the National Patient Demographic Service, which incorporates National Death Registry information as well as local notifications. All patients from the validation cohort were followed up from the time of the procedure until death or censored 30.08.2020.
2.1. Ethics. The current study complies with the Declaration of Helsinki. Following consultation with our local research ethics committee, no informed consent was required as all data were retrospective, anonymized, and part of an ongoing audit.

2.2. Statistics. All continuous variables were tested for normality using the Kolmogorov-Smirnov test. Data are presented as percentages, mean \pm standard deviation (SD), or median (interquartile range). Differences in proportions were tested with Chi-square test or Fisher's exact test, and differences in continuous variables were tested with ANOVA/Student's $t$-test or Kruskal-Wallis/Wilcoxon's signed-rank sum test for parametric and nonparametric variables, respectively.

We deliberately elected to create a 30-day mortality prediction model based on admission information and nonpast medical history, which can often be inaccurate or impossible to obtain at the frontline when patients are confused, in shock, in pain, or intubated and ventilated.

Forward conditional logistic regression analysis was performed to determine the main "frontline" predictors of 30-day mortality. All categorical variables that were significantly different in the two groups (alive vs. deceased at 30 days) and all continuous/ordinal variables that were significantly different $p<0.05$ with an area under the curve AUC $>0.7$ for 30 -day mortality were included in the final binary logistic regression model.

For continuous variables of interest (lactate), we used the Youden's index [10] to identify optimal cutoff points on the receiver operating characteristic (ROC) curve.

The binary logistic regression model (dependent variable 30-day survival) included the following independent variables: age, gender, systolic blood pressure (SBP), ECG changes, bedside LV systolic assessment, arterial lactate, $\mathrm{pH}$, base excess (BE), cardiac arrest, ongoing resuscitation, and intubation-ventilation preprocedure.

The $C$-statistic-related AUC of the models created was compared using the DeLong methodology [11].

The selected variables included in the final model were also included in a Cox-regression model to assess whether they are predictors of long-term mortality.

Based on the binary logistic regression model (Table 1) and currently existing cardiogenic shock protocols, the simplified SAVE (Systolic blood pressure, Arterial blood gas, and left Ventricular Ejection fraction) ACS A, B, C, D, and $\mathrm{E}$ shock groups were defined (Table 2), and their classification power, for 30-day mortality, was tested using ROC curves.

In the validation cohort, we assessed the $C$-statistic of Model 6 (Table 3) and the C-statistic of the SAVE ACS classification in predicting 30 -day mortality.

\section{Results}

During the study period, a total of 11439 index ACS cases that underwent PCI were included in the derivation cohort. Of those, 6039 (52.8\%) were STEMIs, 5392 (47.1\%) were 
TABLE 1: Independent predictors (categorised) of 30-day mortality.

\begin{tabular}{|c|c|c|c|}
\hline Variables & OR & $95 \% \mathrm{CI}$ & $p$ value \\
\hline Age (per 10 years increase) & 1.69 & 1.41 to 2.03 & $<0.001$ \\
\hline $\mathrm{SBP}<90 \mathrm{mmHg}$ & 2.28 & 1.35 to 3.85 & 0.002 \\
\hline Ventilated on arrival & 6.44 & 3.72 to 11.17 & $<0.001$ \\
\hline \multicolumn{4}{|l|}{ LVEF } \\
\hline Normal/mildly impaired & 1 & & \\
\hline Moderately impaired & 1.95 & 1.15 to 3.29 & 0.013 \\
\hline Severely impaired & 2.3 & 1.33 to 3.97 & 0.003 \\
\hline \multicolumn{4}{|l|}{ Lactate $(\mathrm{mmol} / \mathrm{L}) *$} \\
\hline$<2$ & 1 & & \\
\hline $2-5$ & 1.81 & 1.09 to 3.02 & 0.023 \\
\hline$>5$ & 7.2 & 3.63 to 14.34 & $<0.001$ \\
\hline \multicolumn{4}{|l|}{ Base excess tertiles $(\mathrm{mmol} / \mathrm{L})$} \\
\hline-0.4 to +16 & 1 & & \\
\hline-3.9 to -0.5 & 3.67 & 1.66 to 8.12 & 0.001 \\
\hline-28 to -4 & 4.51 & 2.02 to 10.07 & $<0.001$ \\
\hline
\end{tabular}

Model AUC 0.883 , (0.856 to 0.911), $p<0.001$ for death in 30 days and OR: odds ratio, CI: confidence interval, SBP: systolic blood pressure, LVEF: left ventricular ejection fraction, AUC: area under the curve, $*$ the SCAI shock classification lactate cutoffs were used.

TABLE 2: Frontline SAVE ACS classification.

\begin{tabular}{|c|c|c|c|c|c|c|}
\hline & No shock & $\mathrm{A}$ & $\mathrm{B}$ & $\mathrm{C}$ & $\mathrm{D}$ & $\mathrm{E}$ \\
\hline N (\%) & $163(17.5 \%)$ & $98(10.4 \%)$ & $287(30.4 \%)$ & $262(27.7 \%)$ & $109(11.5 \%)$ & $24(2.5 \%)$ \\
\hline SBP & $\geq 90 \mathrm{mmHg}$ & $\geq 90 \mathrm{mmHg}$ & Any & Any & Any & $<90 \mathrm{mmHg}$ \\
\hline LV impairment & Mild & Mod/severe & Mod/severe & Mod/severe & Mod/severe & Severe \\
\hline Lactate & $<2$ & $<2$ & $<2$ & $2-5$ & $>5$ & $>5$ \\
\hline $\mathrm{BE}$ & $>-0.5$ & $>-0.5$ & $<-0.5$ & $<-0.5$ & $<-0.5$ & $<-4$ \\
\hline 30-day mortality (\%) & 1.2 & 4.1 & 13.6 & 29.4 & 64.2 & 87.5 \\
\hline 30-day mortality (\%) if not intubated & 0.7 & 2.3 & 11.2 & 23.4 & 55.6 & 62.5 \\
\hline
\end{tabular}

Data from 943 patients classified according to the SAVE criteria, AUC for 30-day mortality 0.814 ( 0.782 to 0.845 ), $p<0.001$, SBP: systolic blood pressure, LV: left ventricle, BE: base excess, and AUC: area under the curve.

TABLE 3: Forward conditional logistic regression analysis determining main independent "frontline" predictors of 30-day mortality.

\begin{tabular}{|c|c|c|c|c|c|}
\hline & & OR (95\% CI) & $p$ value & AUC $_{C \text {-statistic }}$ & $p$ value \\
\hline Model 1 & Lactate & 1.55 (1.42 to 1.69$)$ & $<0.001$ & 0.787 (0.745 to 0.828$)$ & $<0.001$ \\
\hline \multirow{2}{*}{ Model 2} & Lactate & $1.46(1.34$ to 1.6$)$ & $<0.001$ & $0.830 *$ & $<0.001$ \\
\hline & Intubated & 6.09 (3.9 to 9.6$)$ & $<0.001$ & 0.793 to 0.867$)$ & \\
\hline \multirow{3}{*}{ Model 3} & Lactate & 1.48 (1.35 to 1.62$)$ & $<0.001$ & \multirow{3}{*}{$0.858 *(0.825$ to 0.891$)$} & \multirow{3}{*}{$<0.001$} \\
\hline & Intubated & $9.74(5.8$ to 16.33$)$ & $<0.001$ & & \\
\hline & Age & $1.06(1.04$ to 1.08$)$ & $<0.001$ & & \\
\hline \multirow{4}{*}{ Model 4} & Lactate & $1.28(1.15$ to 1.43$)$ & $<0.001$ & \multirow{4}{*}{$0.868 *(0.837$ to 0.900$)$} & \multirow{4}{*}{$<0.001$} \\
\hline & Intubated & 9.35 (5.55 to 15.74$)$ & $<0.001$ & & \\
\hline & Age & $1.06(1.04$ to 1.08$)$ & $<0.001$ & & \\
\hline & $\mathrm{BE}$ & $1.11(1.05$ to 1.17$)$ & $<0.001$ & & \\
\hline \multirow{7}{*}{ Model 5} & Lactate & $1.27(1.13$ to 1.42$)$ & $<0.001$ & \multirow{7}{*}{$0.878(0.850$ to 0.907$)$} & \multirow{7}{*}{$<0.001$} \\
\hline & Intubated & 78.34 (4.89 to 14.2$)$ & $<0.001$ & & \\
\hline & Age & $1.05(1.04$ to 1.07$)$ & $<0.001$ & & \\
\hline & $\mathrm{BE}$ & $1.11(1.05$ to 1.17$)$ & $<0.001$ & & \\
\hline & LVEF-mild/N & REF & & & \\
\hline & LVEF-mod & $1.88(1.21$ to 3.16$)$ & 0.017 & & \\
\hline & LVEF-severe & $2.18(1.28$ to 3.75$)$ & 0.004 & & \\
\hline \multirow{8}{*}{ Model 6} & Lactate & $1.25(1.12$ to 1.41$)$ & $<0.001$ & \multirow{8}{*}{$0.879(0.851$ to 0.908$)$} & \multirow{8}{*}{$<0.001$} \\
\hline & Intubated & $7.4(4.3$ to 12.74$)$ & $<0.001$ & & \\
\hline & Age & 1.05 (1.04 to 1.07$)$ & $<0.001$ & & \\
\hline & $\mathrm{BE}$ & $1.10(1.04$ to 1.17$)$ & $<0.001$ & & \\
\hline & LVEF-mild/N & REF & & & \\
\hline & LVEF-mod & $1.84(1.09$ to 3.1$)$ & 0.022 & & \\
\hline & LVEF-severe & $1.98(1.14$ to 3.42$)$ & 0.015 & & \\
\hline & SBP & $0.99(0.982$ to 0.999$)$ & 0.024 & & \\
\hline
\end{tabular}

$N=892$ patients included in the analysis. J: variables included in the regression model: age, gender, cardiac arrest, ongoing resuscitation on admission, ECG on admission, intubated/ventilated on admission, LVEF category, SBP, pH, lactate, and BE, * statistically significant increase in the AUC when compared to previous model, OR: odds ratio, CI: confidence interval, AUC: area under the curve, BE: base excess, LVEF: left ventricular ejection fraction, SBP: systolic blood pressure, and ECG: electrocardiogram. 
NSTEMIs, and $8(0.1 \%)$ unstable angina cases. Mean age was $64.1 \pm 14.5$ years and $8280(72.4 \%)$ were male.

Table 4 shows the differences in demographics and frontline data based on vitality status at 30 days. Patients who subsequently died in 30 days were older, most commonly females, with a higher prevalence of clinical hypoperfusion and pulmonary oedema on arrival, lower systolic blood pressure, and a higher incidence of ECG changes (particularly T-wave inversion and ST depression)

A total of 3752 patients $(32.8 \%)$ had arterial blood gas $(\mathrm{ABG})$ prior to any intervention due to concerns raised in clinical assessment (Table 4). Indeed, patients who had their lactate measured had significantly higher 30-day mortality compared to those who did not have an $\mathrm{ABG}(17.1 \%$ vs. $3.3 \%, p<0.001)$

In patients who had their lactate measured, 30-day mortality AUC for admission lactate was 0.780 (0.759 to 0.801) $(p<0.001)$. Applying the Youden index, the optimal lactate cutoff value to predict 30-day mortality was 2.65 with a sensitivity of 0.646 and a specificity of 0.796 .

Patients who were deceased at 30 days exhibited significantly worse LV function on admission on bedside echocardiography. Cardiac arrest was present in more than half (51.4\%) of patients who subsequently died within 30 days, whereas nearly a third (32.1\%) were intubated on arrival. Asystole and electromechanical dissociation (EMD) were arrest rhythms that were most likely to predict death at 30 days.

In Table 5, we present the past medical history and procedural data based on vitality status at 30 days.

Figure 1 demonstrates the presence of hypoperfusion (lactate $>2 \mathrm{mmol} / \mathrm{L}$ ) in a third of normotensive ACS patients (29.2\% of patients with SBP $>90 \mathrm{mmHg}$ have a lactate between 2 and $5 \mathrm{mmol} / \mathrm{L}$, whereas $5.7 \%$ of normotensive patients have a lactate $>5 \mathrm{mmol} / \mathrm{L})$. Even when excluding patients with resuscitated cardiac arrest, $30 \%$ of normotensive ACS present with a lactate of $>2 \mathrm{mmol} / \mathrm{L}$.

When examining hypoperfused patients (defined as lactate $>2 \mathrm{mmol} / \mathrm{L}), 78 \%$ had SBP $>90 \mathrm{mmHg}$.

3.1. Multivariable Analysis. A total of 892 patients were included in the multivariable analysis (full set of data) as shown in Table 3.

Independent predictors of 30-day mortality by order of significance were lactate, intubation/ventilation on arrival, age, BE, LVEF category, and SBP. The different models and corresponding $C$-statistics are shown in Table 3. In Model 6, an AUC of 0.879 (0.851 to 0.908) was achieved, which suggests a very good performance (Figure 2). As noted, the contribution of SBP to the incremental value of the $C$-statistic is rather small, and no statistically significant difference was seen when comparing the $C$-statistic of Models 5 and 6 .

In Supplementary Table 1, all variables included in Model 6, except for SBP, predicted long-term survival.

When using the same variables but dichotomized as per established criteria [7] (e.g., SBP $<90 \mathrm{mmHg}$, lactate cutoff values of 2 and 5(7), and BE tertiles), the model discrimination remains excellent with an AUC of 0.883 for 30-day mortality (Table 1).
In the validation cohort, Model 6 variables demonstrated an excellent discrimination for 30-day mortality with a $C$ statistic of $0.949(0.887-1)(p<0.001)$.

Frontline SAVE (systolic blood pressure, arterial blood gas, and left ventricular ejection fraction) ACS shock classification: the frontline shock classification includes only 4 variables readily available within a short time frame from admission; arterial SBP, LV function, lactate, and BE. Since age and intubation, even though strong predictors of mortality are not indicative of the hemodynamic status of the patients, we elected to use the 4 remaining variables (from Table 1) to classify patients in the following subgroups (no shock, A, B, C, D, and E). Each group can subsequently predict 30-day and long-term mortality (Table 2, Figure 3).

It should be noted that this classification relies more on the variables that were shown to significantly contribute to the forward conditional regression analysis from Table 3. Less importance was given to systolic blood pressure in defining shock as it contributed nonsignificant increments in AUC in Model 6 (Table 3).

The SAVE ACS classification AUC for 30-day mortality was $0.814(0.782$ to $0.845, p<0.001)$, suggestive of a very good discrimination. Furthermore, the SAVE ACS classification predicted long-term survival as shown in Figure 3 $\left(p_{\text {log-rank }}<0.001\right)$.

The discrimination of the SAVE ACS classification in the validation cohort was very good with a $C$-statistic of 0.815 ( 0.725 to $0.905, p<0.001)$

Based on the SAVE ACS classification, the authors created an algorithm for the use of MCS in the patient group with the highest 30-day mortality and biochemical/echocardiographic indices compatible with classic or worsening shock (Figure 4). Prior to any decision for MCS, the shock team would ensure there are no contraindications (Supplementary Figure (available here)).

\section{Discussion}

In the current retrospective study, we have identified 5 variables apart from age, that are readily available to frontline staff (SBP, LV function, intubation, arterial lactate, and base excess) that can help identify rapidly those ACS patients at higher risk of death at 30 days. These variables alone showed an excellent discriminatory power for predicting 30-day mortality with an AUC of 0.883 in the derivation and 0.949 in the validation cohort. Based on these variables, we introduced the "SAVE ACS" classification, which can be rapidly carried out by frontline staff to classify ACS patients in different stages of CS. This would allow physicians to make informed decisions on early MCS use in patients with a higher 30-day mortality risk (Figure 4). The current study also highlights the important concept of "normotensive shock," originally introduced by Menon et al. [12] in 2000, as nearly $80 \%$ of hypoperfused patients (defined as lactate $>2 \mathrm{mmol} / \mathrm{L}$ ) were shown to have a SBP $>90 \mathrm{mmHg}$ (Figure 1).

CS definitions have been very variable across different studies. Few decades ago, Forrester et al. in their classification [13] using right-sided heart catheterization described 
TABLE 4: Frontline variables in ACS patients grouped by vital status at 30 days.

\begin{tabular}{|c|c|c|c|c|}
\hline & \multicolumn{2}{|c|}{ Survival at 30 days } & \multirow{2}{*}{$p$ value } & \multirow{2}{*}{ AUC } \\
\hline & Alive $(N=10548)$ & Deceased $(N=891)$ & & \\
\hline \multicolumn{5}{|l|}{ Demographics $(N=11439)$} \\
\hline Age (years) & $63.4 \pm 14.2$ & $72.6 \pm 14.0$ & $<0.001$ & \\
\hline Gender (male) $N(\%)$ & $7687(72.9)$ & $593(66.6 \%)$ & $<0.001$ & \\
\hline \multicolumn{5}{|l|}{ Clinical assessment $(N=10107)$} \\
\hline Killip class $(\%)$ & & & & $0.675(0.651-0.698)^{*}$ \\
\hline (I) No evidence of heart failure & 91.5 & 58.1 & & \\
\hline (II) Basal crepitations/raised venous pressure & 4.5 & 9.2 & & \\
\hline (III) Pulmonary oedema & 2.6 & 8.8 & $<0.001$ & \\
\hline (IV) Cardiogenic shock & 1.4 & 23.9 & & \\
\hline \multicolumn{5}{|l|}{ Baseline blood pressure $(N=6110)$} \\
\hline Systolic blood pressure $(\mathrm{mmHg})$ & $129.2 \pm 25.2$ & $98 \pm 36.2$ & $<0.001$ & $0.770(0.742-0.798)^{*}$ \\
\hline Diastolic blood pressure (mmHg) & $77.9 \pm 16.9$ & $79.2 \pm 29.8$ & 0.411 & \\
\hline Inotropic support on arrival $N(\%)$ & $72(0.7)$ & $57(6.4)$ & $<0.001$ & \\
\hline \multicolumn{5}{|l|}{ Electrocardiogram on admission $(N=11189)(\%)$} \\
\hline No dynamic changes & 10.6 & 7.7 & & \\
\hline LBBB & 4.9 & 6.1 & & \\
\hline Other abnormality & 5.2 & 9.7 & & \\
\hline T-wave changes & 7.4 & 2.6 & $<0.001$ & \\
\hline ST depression & 9 & 11.7 & & \\
\hline ST segment elevation & 63 & 62.2 & & \\
\hline \multicolumn{5}{|l|}{ Bedside LV systolic function assessment $(N=4448)(\%)$} \\
\hline Good/mildly impaired (>45\%) & 70.3 & 30.7 & & \\
\hline Moderate impairment (35-45\%) & 20.4 & 27.6 & $<0.001$ & $0.728(0.700-0.756)^{*}$ \\
\hline Severely impaired $(<35 \%)$ & 9.3 & 41.7 & & \\
\hline \multicolumn{5}{|l|}{ Arterial blood gas on admission $(N=3752)$} \\
\hline Lactate $(\mathrm{mmol} / \mathrm{L})$ & $1.5(1.1$ to 2.4$)$ & 3.7 (2.0 to 7.1$)$ & $<0.001$ & $0.780(0.759-0.801)^{*}$ \\
\hline $\mathrm{pH}$ & $7.41(7.37$ to 7.45$)$ & $7.31(7.24$ to 7.39$)$ & $<0.001$ & $0.773(0.743-0.803)^{*}$ \\
\hline $\mathrm{pO} 2(\mathrm{kPa})$ & $11.3(9.11$ to 14.2$)$ & $12.55(9.35$ to 21.45$)$ & $<0.001$ & $0.559(0.532-0.586)^{*}$ \\
\hline $\mathrm{pCO} 2(\mathrm{kPa})$ & $4.92(4.35$ to 5.5$)$ & $5.29(4.37$ to 6.27$)$ & $<0.001$ & $0.580(0.552-0.609)^{*}$ \\
\hline $\mathrm{BE}(\mathrm{mmol} / \mathrm{L})$ & $-1.2(-3.6$ to 1$)$ & $-6.35(-8.9$ to 2.8$)$ & $<0.001$ & $0.778(0.756-0.800)^{*}$ \\
\hline $\mathrm{K}(\mathrm{mmol} / \mathrm{L})$ & $3.8(3.5$ to 4.2$)$ & $4.1(3.7$ to 4.6$)$ & $<0.001$ & $0.605(0.578-0.632)^{*}$ \\
\hline $\mathrm{Glc}(\mathrm{mmol} / \mathrm{L})$ & $7.6(6.3$ to 10.1$)$ & $10.6(7.78$ to 14.8$)$ & $<0.001$ & $0.693(0.670-0.717)^{*}$ \\
\hline \multicolumn{5}{|l|}{ Cardiac arrest parameters $(N=11439)$} \\
\hline Cardiac arrest $N(\%)$ & $816(7.7)$ & $458(51.4)$ & $<0.001$ & \\
\hline Cardiac arrest on admission-ongoing resuscitation $N(\%)$ & $6(0.1)$ & $29(3.3)$ & $<0.001$ & \\
\hline Ventilated preprocedure $N(\%)$ & $215(2.1)$ & $273(32.1)$ & $<0.001$ & \\
\hline Type of arrest & & & $<0.001$ & \\
\hline Asystole & 8.2 & 18.2 & & \\
\hline EMD & 2.8 & 21.1 & & \\
\hline $\mathrm{VF} /$ pulseless VT & 79.2 & 58.1 & & \\
\hline Unknown & 9.8 & 2.6 & & \\
\hline
\end{tabular}

AUC: area under the curve, LBBB: left bundle branch block, LV: left ventricle, BE: base excess, Glc: glucose, EMD: electromechanical dissociation, VF: ventricular fibrillation, and VT: ventricular tachycardia.

the role of cardiac hemodynamics in stratifying the risk after acute MI in the pre-PPCI era. Patients in Forrester et al.'s subgroup IV with a pulmonary capillary wedge pressure (PCWP) $>18 \mathrm{~mm} \mathrm{Hg}$ and a cardiac index (CI) $<2.2 \mathrm{~L} \cdot \mathrm{min}^{-1} \cdot \mathrm{m}^{-2}$, indicative of CS, had a mortality of $51 \%$. In the ongoing DANGER trial, tissue hypoperfusion was defined as lactate $\geq 2.5 \mathrm{mmol} / \mathrm{L}$, persistent hypotension with $\mathrm{SBP}<100 \mathrm{mmHg}$, and/or need for inotropes and LV $<45 \%$ on echocardiography [14]. In the anticipated ECLS-shock trial [15], the entry criteria include, amongst others, $\mathrm{SBP}<90 \mathrm{mmHg}$ for $>30 \mathrm{~min}$ or catecholamines to maintain pressure $>90 \mathrm{mmHg}$ and an arterial lactate of $>3 \mathrm{mmol} / \mathrm{L}$. In another large trial [16], the EURO Shock randomized study entry criteria include yet again hypotension defined as
$\mathrm{SBP}<90 \mathrm{mmHg}$ for at least $30 \mathrm{~min}$ or inotropic support to maintain a SBP $>90 \mathrm{mmHg}$ and organ hypoperfusion measured as lactate $>2 \mathrm{mmoL}$. One common theme in these studies is the difficulty in recruitment, which can be explained by the exclusion of patients with normotensive hypoperfusion, who constitute a third of ACS patients. As shown in Figure 1, in our cohort, the majority $(78 \%)$ of hypoperfused patients (lactate $>2 \mathrm{mmol} / \mathrm{L}$ ) have SBP over $90 \mathrm{mmHg}$. It may also well be that patients who are both hypoperfused and hypotensive are the ones far too deep in the spiral of cardiogenic shock, with futile outcomes despite MCS deployment.

Baran et al. [7] identified these differences in classifying shock in ongoing clinical trials and published a 
TABLE 5: Past medical history and procedural data grouped by vital status at 30 days.

\begin{tabular}{|c|c|c|c|}
\hline & \multicolumn{2}{|c|}{ Survival at 30 days } & \multirow[b]{2}{*}{$p$ value } \\
\hline & $\begin{array}{c}\text { Alive } \\
(N=10548)\end{array}$ & $\begin{array}{l}\text { Deceased } \\
(N=891)\end{array}$ & \\
\hline \multicolumn{4}{|l|}{ Past medical history } \\
\hline Previous MI N (\%) & $1679(16.1)$ & $163(18.7)$ & 0.045 \\
\hline $\begin{array}{l}\text { Previous CABG } n \\
(\%)\end{array}$ & $553(5.3)$ & $80(9.2)$ & $<0.001$ \\
\hline Previous PCI N (\%) & $1311(12.6)$ & $83(9.5)$ & 0.008 \\
\hline Diabetes $N(\%)$ & $2020(19.9)$ & $198(23.5)$ & 0.014 \\
\hline Hypertension $N(\%)$ & $4347(41.2)$ & $344(38.6)$ & 0.129 \\
\hline Total cholesterol & $5.34 \pm 16.2$ & $5.1 \pm 1.5$ & 0.916 \\
\hline $\begin{array}{l}\text { Asthma/COPD N } \\
(\%)\end{array}$ & $682(6.6)$ & $88(10.4)$ & $<0.001$ \\
\hline CVA $N(\%)$ & $85(0.8)$ & $12(1.3)$ & 0.091 \\
\hline Creatinine & $87.5 \pm 1.36$ & $131.3 \pm 5.49$ & $<0.001$ \\
\hline \multicolumn{4}{|l|}{ Smoking status (\%) } \\
\hline Never smoked & 43.4 & 48.5 & \multirow{3}{*}{$<0.001$} \\
\hline Ex-smoker & 27.4 & 31.2 & \\
\hline Current smoker & 29.1 & 20.3 & \\
\hline \multicolumn{4}{|l|}{ Procedural data } \\
\hline LMS disease $N(\%)$ & $301(4.4)$ & $93(20)$ & $<0.001$ \\
\hline \multicolumn{4}{|l|}{$\begin{array}{l}\text { Epicardial disease } N \\
\text { (\%) }\end{array}$} \\
\hline Single-vessel & $4226(63.2)$ & $195(44.6)$ & \multirow{3}{*}{$<0.001$} \\
\hline Two-vessel & $1632(24.4)$ & $138(31.6)$ & \\
\hline Three-vessel & $626(9.4)$ & $95(21.7)$ & \\
\hline Inotropes $N(\%)$ & $72(0.7)$ & $57(6.4)$ & $<0.001$ \\
\hline IABP $\mathrm{n}(\%)$ & $213(2)$ & $96(10.8)$ & $<0.001$ \\
\hline Impella $* N(\%)$ & $7(0.1)$ & $9(1)$ & $<0.001$ \\
\hline $\mathrm{ECMO} * N(\%)$ & $8(0.1)$ & $20(2.2)$ & $<0.001$ \\
\hline \multicolumn{4}{|l|}{$\begin{array}{l}\text { TIMI flow after PCI } \\
\text { (\%) }\end{array}$} \\
\hline 0 & 2.1 & 7.7 & \multirow{4}{*}{$<0.001$} \\
\hline I & 0.9 & 4.1 & \\
\hline II & 3.7 & 12.3 & \\
\hline III & 93.2 & 75.9 & \\
\hline \multicolumn{4}{|l|}{ Access site (\%) } \\
\hline Radial & 63.9 & 35.9 & \multirow{2}{*}{$<0.001$} \\
\hline Femoral & 36.1 & 64.1 & \\
\hline
\end{tabular}

* ECMO and Impella entry in database 2018 onwards, MI: myocardial infarction, CABG: coronary artery bypass surgery, PCI: percutaneous coronary intervention, COPD, chronic obstructive pulmonary disease, CVA: cerebrovascular accident, LMS: left main stem disease, IABP: intraaortic balloon pump, ECMO: extra corporeal membrane oxygenation, and TIMI: thrombolysis in myocardial infarction.

comprehensive consensus on shock classification that would unify the scientific community and lead to more reproducible outcomes. One of the well-thought clauses in the SCAI shock classification for the hemodynamic definition of shock is " $>30 \mathrm{mmHg}$ drop from baseline SBP." This, however, takes for granted the knowledge of the patient's normal baseline SBP, which is often an unknown parameter. Hence, the definition of shock based on SBP drop from baseline can be challenging. Invasive hemodynamics, even though desirable for established intensive care patients, cannot be readily obtained in the primary PCI setting where shorter door-to-balloon [17] times have been associated with improved future survival. Door-to-unload [18] time is also
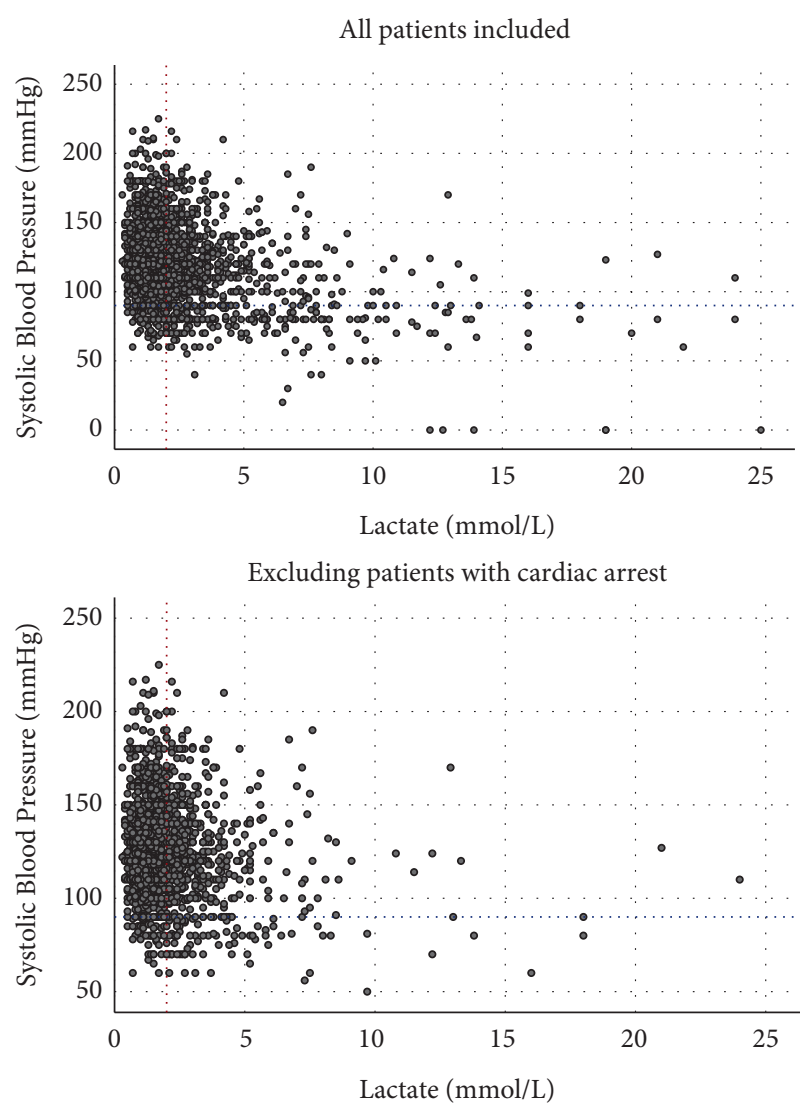

FIGURE 1: Scatter plots of lactate and systolic blood pressure (SBP) of patients presenting with acute coronary syndromes. In the top panel, all patients are included, whereas in the bottom one, patients with previous or ongoing cardiac arrest are excluded. The red dotted line indicates lactate cutoff of $2 \mathrm{mmol} / \mathrm{L}$, whereas the blue dotted line indicates the traditional $90 \mathrm{mmHg}$ SBP.

emerging as an important concept and delay in shock classification and timely MCS deployment may have important implications in survival. Hence, studies that advocate physicians to wait for $30 \mathrm{~min}[15,16]$ to assess the presence of shock may be allowing patients to drift deeper into the spiral of shock, depriving them of the benefit of early MCS [19]. Of note, in our dataset, only $15 \%$ of patients with lactate between 2 and $5 \mathrm{mmol} / \mathrm{L}$ had a SBP $<90 \mathrm{mmHg}$, whereas of those with a lactate $>5 \mathrm{mmol} / \mathrm{L}$, only $45 \%$ had a SPB $<90 \mathrm{mmHg}$. This suggests that studies advocating a $90 \mathrm{mmHg}$ cutoff for the definition of shock are excluding a vast number of patients who have already exhibited tissue hypoperfusion.

In a multivariable analysis of the FRENCHSHOCK registry $(N=772)$ of patients admitted to ITU with cardiogenic shock [20] (albeit nonischemic in 64\%), independent predictors of 30-day mortality included age, low SBP, high arterial lactate, low eGFR, and low LVEF. These results broadly match our set of variables even though our data relates to patients presenting with ACS. The similarity of these studies suggests that cardiogenic shock of any aetiology has similar predictors, assuming attempts for effective revascularization have occurred where indicated. 


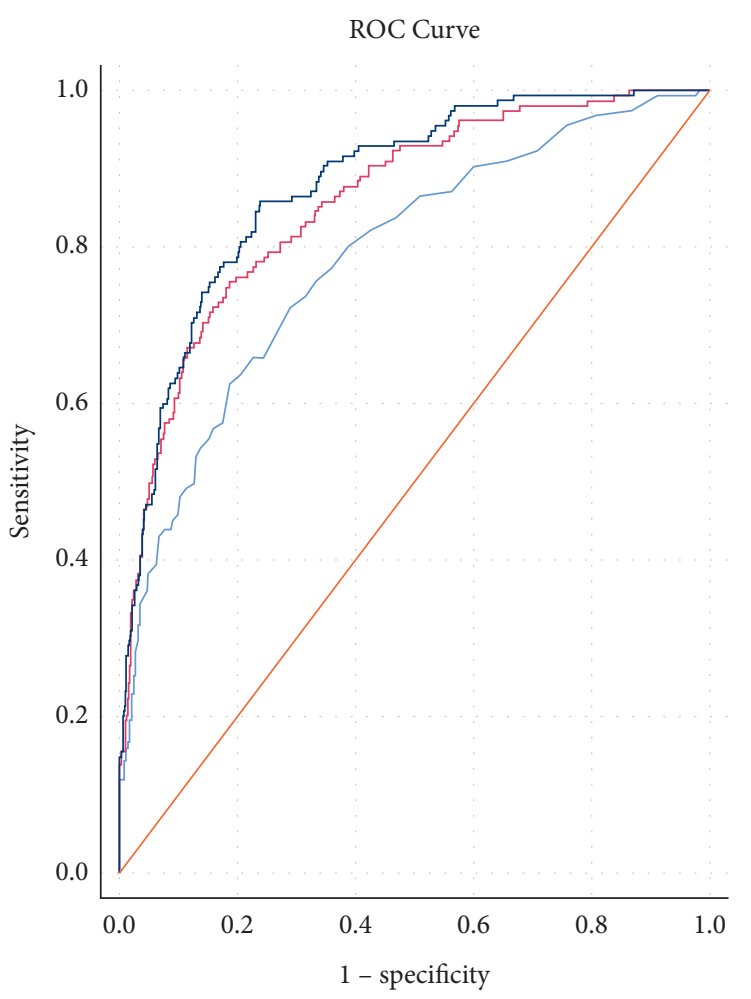

Source of the Curve

$$
\begin{array}{ll}
- & \text { Model } 1 \\
- & \text { Model } 3 \\
- & \text { Model } 6 \\
- & \text { Reference line }
\end{array}
$$

FIgURE 2: Comparison of the receiver operating characteristic (ROC) curves of different regression models predicting 30-day mortality. Model 1: Lactate. Model 3: lactate, intubation, age. Model 6: lactate, intubation, age, base excess, left ventricular ejection fraction, and systolic blood pressure.

Furthermore, in line with our findings, a study of 165 ischemic cardiogenic shock patients identified baseline serum bicarbonate values to be predictive of 28-day mortality [9]. Metabolic acidosis is a multifactorial event caused by the combination of bicarbonate loss by the kidneys, mounting ketones, accumulation of inorganic acids, and systemic lactic acidosis [21]. Given that acute kidney injury emerges early in patients with CS [22], it is possible that in patients with impending CS, metabolic acidosis ensues first in the pathophysiological cascade, tagging the patients at risk (group B in the SAVE ACS classification). Furthermore, it has been established that acidosis reduces cardiac contractility and enhances vascular hyporesponsiveness to vasopressors, precipitating a vicious circle leading to worsening CS [21].

The importance of arterial lactate value in predicting short-term mortality has been identified in several studies. In patients with septic shock, a presenting blood lactate of more than $2.5 \mathrm{mmol} / \mathrm{L}$ showed the best discrimination (the largest area under the ROC curve to predict 28-day mortality of 0.7 ) [23]. A systematic review of 33 studies [24] concluded that

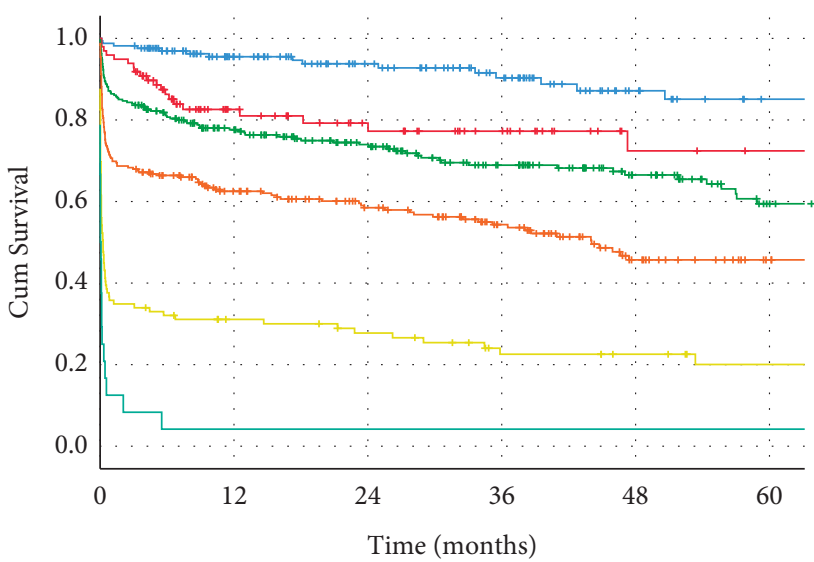

SAVE group

$\neg$ No shock

- No shock-censored

$\neg \mathrm{A}$

$\neg \mathrm{B}$

$\neg \mathrm{C}$

$\longrightarrow \mathrm{D}$

$\neg \mathrm{E}$

+ A-censored

+ B-censored

+ C-censored

- D-censored

- E-censored

\begin{tabular}{|l|c|c|c|}
\hline Months of FU & 0 & 12 & 60 \\
\hline No shock & 165 & 121 & 34 \\
\hline A & 98 & 53 & 13 \\
\hline B & 287 & 186 & 45 \\
\hline C & 262 & 136 & 27 \\
\hline D & 109 & 29 & 8 \\
\hline E & 24 & 1 & 0 \\
\hline
\end{tabular}

FIGURE 3: Kaplan-Meier curves showing 5-year survival of patients in different stages of shock, as per SAVE ACS classification.

patients presenting to the hospital with a lactate of more than $2.5 \mathrm{mmol} / \mathrm{L}$ on admission should be closely monitored for signs of deterioration. Another recent study [25] on an unselected A\&E population of 14015 patients, revealed an optimal lactate cutoff (using also Youden's index) of 2.6 and an AUC of 0.711 . The 30-day mortality in those with high lactate was $20.8 \%$ versus $6.5 \%$ in those in the lower lactate group. Interestingly, the ongoing DANGER trial also uses a lactate cutoff of 2.5 based on a retrospective study of 2094 suspected STEMI patients [26]. In the latter study [26], the use of SBP, LVEF, and lactate as continuous variables led to an AUC of $0.88(p<0.001)$ for prediction of 30 -day mortality, highlighting the importance of including lactate and echocardiographic LV assessment in the definition of shock. In our data, a lactate value of 2.65 was the best discriminator of 30-day mortality and appears to be in agreement with all aforementioned studies.

In patients with MI-related shock, an algorithm from the IABP-SHOCK II trial was produced to predict mortality [27]. These predictors included age $>73$ (1 point), prior stroke ( 2 points), glucose on admission $>10.6 \mathrm{mmol} / \mathrm{L}$ (1 point), creatinine on admission $>132.6$ (1 point), TIMI flow grade $<3$ after PCI ( 2 points), and blood lactate on admission of $>5 \mathrm{mmol} / \mathrm{L}$ ( 2 points). Of interest, the predictive value of this score had an AUC of 0.74, which is lower than that the 


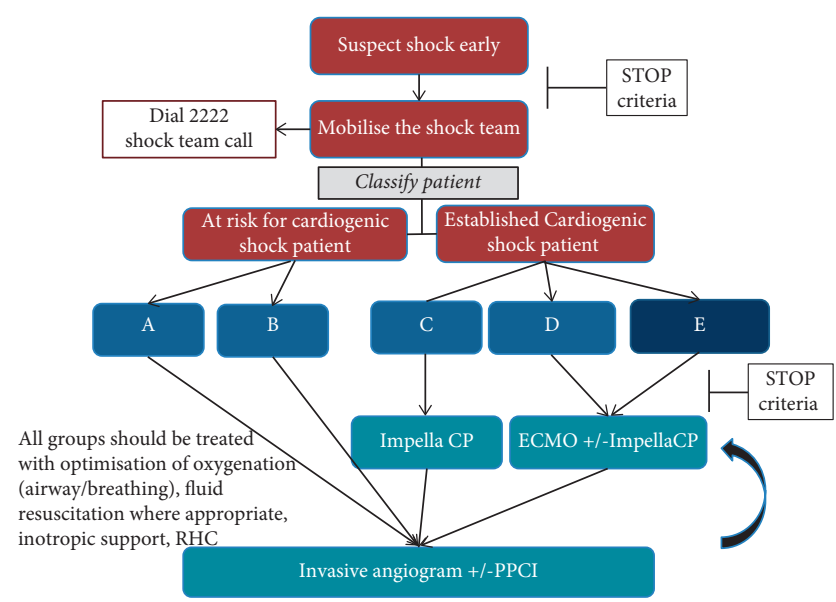

FIGURE 4: Proposed treatment algorithm for patients presenting with acute coronary syndromes and shock. Early use of mechanical circulatory support is advocated for patients in the C-E groups.

one seen in our model or indeed the one produced by Frydland et al. using only three variables [26]. Furthermore, creatinine is not always readily available on admission nor is the TIMI flow after PCI. Therefore, this algorithm is not fit for this purpose, as it cannot rapidly classify patients into shock groups who may be in need of early MCS before PCI. Similarly, the Global Registry of Acute Coronary Events (GRACE) score [28] was developed for patients presenting with ACS. The GRACE score predicts 6-month and longerterm mortality [29] with $C$-statistics in the 0.8 mark. The GRACE score was later slightly modified by Fox et al. [30] using a cohort of 43810 patients. Of interest, perfusion indices, such as lactate, are not included in the GRACE score, whereas "creatinine" and "troponin," which are amongst the 8 variables required to calculate the score, are not always readily available when performing an initial assessment of a patient with ACS.

In the current study, we aimed to highlight that in patients presenting with ACS, CS is a continuum and traditional definitions with arbitrary cutoffs of SBP $<90 \mathrm{mmHg}$ have to be replaced by more robust, yet easy-to-use, classifications that reflect the severity of tissue hypoperfusion. Patients admitted with ACS are often in pain and emotional distress, leading to increased sympathoadrenal activation [23]. Hence, it is not uncommon that patients with tissue hypoperfusion present with a systolic blood pressure over $90 \mathrm{mmHg}$, driven purely by the activation of their sympathetic system. Once the stressor is removed (revascularization), coupled with reperfusion [31], the blood pressure starts to drift downwards unmasking the underlying hemodynamic compromise. In such cases, echocardiography, metabolic acidosis, and raised lactate can identify the underlying impending doom at an earlier stage, thus allowing physicians to act faster, preventing the patient from sliding into the irreversible part of the CS spiral.

This, as every retrospective study, is not without its limitations. Our classification was created based on patients who had a full set of variables (selection bias). These were, invariably, patients at the sicker end of the spectrum as demonstrated by the significantly higher 30-day mortality of those who had lactate measured. They, however, are also the patients who would benefit the most from a rapid shock classification and MCS deployment. Heart rate at presentation was not accurately recorded; hence, it was not included in our models. The predictive power of our classification was confirmed in the validation cohort from our institution. Furthermore, we would like to highlight that this classification was derived from ACS patients that underwent PCI, and it excluded those who had normal coronaries, those that underwent emergency coronary artery bypass, or indeed those in whom any intervention was deemed futile. Collaborations with other centers have been set in motion to validate our findings in large independent ACS cohorts. Figure 4 is conceptual and not supported by any data in this or other studies. It is, however, proposing an escalating level of MCS depending on the level of CS the patient is in, with the least invasive option (Impella CP) for the early/classic stages of shock (C) and the use of ECMO in combination with Impella for those in deeper shock (D and E).

A future randomized trial assessing the benefit of early MCS use in SAVE ACS groups C, D, E, and potentially B would allow us to draw conclusions on the benefits of MCS in these groups.

\section{Conclusions}

The current study describes an easy, rapid classification of CS using variables readily available to frontline physicians. The SAVE ACS aims to complement rather than replace the comprehensive, established SCAI classification. If validated in larger cohorts, the SAVE ACS classification could form the substrate of future randomized trials of early MCS in ACS CS patients.

\section{Data Availability}

Data are available from the corresponding author upon request.

\section{Conflicts of Interest}

Vasileios Panoulas is in receipt of honoraria and consultancy fees from Abiomed. Charles Ilsley has no conflicts of interest to declare.

\section{Acknowledgments}

The authors are grateful to the Catheterization Laboratory Team and Cardiology Department at Harefield Hospital who are tirelessly looking after patients with acute coronary syndromes 24/7 andthe support from the Cardiogenic Shock Fund, Royal Brompton and Harefield Hospitals Charity.

\section{Supplementary Materials}

Supplementary Table 1. Cox-regression analysis for long-term all-cause mortality. Supplementary Figure. Relative 
contraindications to mechanical circulatory support. (Supplementary Materials)

\section{References}

[1] K. S. Rathod, S. Koganti, M. B. Iqbal et al., "Contemporary trends in cardiogenic shock: incidence, intra-aortic balloon pump utilisation and outcomes from the London heart attack group," European Heart Journal: Acute Cardiovascular Care, vol. 7, no. 1, pp. 16-27, 2018.

[2] H. Thiele, E. M. Ohman, S. de Waha-Thiele, U. Zeymer, and S. Desch, "Management of cardiogenic shock complicating myocardial infarction: an update 2019," European Heart Journal, vol. 40, no. 32, pp. 2671-2683, 2019.

[3] J. S. Hochman, C. E. Buller, L. A. Sleeper et al., "Cardiogenic shock complicating acute myocardial infarction-etiologies, management and outcome: a report from the SHOCK Trial Registry," Journal of the American College of Cardiology, vol. 36, no. 3, pp. 1063-1070, 2000.

[4] S. van Diepen, J. N. Katz, N. M. Albert et al., "Contemporary management of cardiogenic shock: a scientific statement from the American heart association," Circulation, vol. 136, pp. e232-e268, 2017.

[5] H. Thiele, U. Zeymer, F. J. Neumann et al., "Intraaortic balloon support for myocardial infarction with cardiogenic shock," New England Journal of Medicine, vol. 367, no. 14, pp. 1287-1296, 2012.

[6] H. Thiele, U. Zeymer, and N. Thelemann, "Intraaortic balloon pump in cardiogenic shock complicating acute myocardial infarction: long-term 6year outcome of the randomized IABP-SHOCK II trial," Circulation, vol. 139, no. 3, pp. 395403, 2018.

[7] D. A. Baran, C. L. Grines, and S. Bailey, "SCAI clinical expert consensus statement on the classification of cardiogenic shock: this document was endorsed by the American college of cardiology (ACC), the american heart association (AHA), the society of critical care medicine (SCCM), and the society of thoracic surgeons (STS) in April 2019," Catheterization Cardiovascular Intervention, vol. 94, no. 1, pp. 29-37, 2019.

[8] M. E. Hands, J. D. Rutherford, J. E. Muller et al., "The inhospital development of cardiogenic shock after myocardial infarction: incidence, predictors of occurrence, outcome and prognostic factors," Journal of the American College of Cardiology, vol. 14, no. 1, pp. 40-46, 1989, discussion 47-8.

[9] O. Wigger, S. Bloechlinger, D. Berger et al., "Baseline serum bicarbonate levels independently predict short-term mortality in critically ill patients with ischaemic cardiogenic shock," European Heart Journal: Acute Cardiovascular Care, vol. 7, no. 1, pp. 45-52, 2018.

[10] W. J. Youden, "Index for rating diagnostic tests," Cancer, vol. 3, no. 1, pp. 32-35, 1950.

[11] E. R. DeLong, D. M. DeLong, and D. L. Clarke-Pearson, "Comparing the areas under two or more correlated receiver operating characteristic curves: a nonparametric approach," Biometrics, vol. 44, no. 3, pp. 837-845, 1988.

[12] V. Menon, J. N. Slater, H. D. White, L. A. Sleeper, T. Cocke, and J. S. Hochman, "Acute myocardial infarction complicated by systemic hypoperfusion without hypotension: report of the SHOCK trial registry," The American Journal of Medicine, vol. 108, no. 5, pp. 374-380, 2000.

[13] J. S. Forrester, G. Diamond, K. Chatterjee, and H. J. C. Swan, "Medical therapy of acute myocardial infarction by application of hemodynamic subsets," New England Journal of Medicine, vol. 295, no. 25, pp. 1404-1413, 1976.
[14] N. J. Udesen, J. E. Møller, M. G. Lindholm et al., "Rationale and design of danger shock: danish-german cardiogenic shock trial," American Heart Journal, vol. 214, pp. 60-68, 2019.

[15] https://clinicaltrials.gov/ct2/show/NCT03637205 Extracorporeal Life Support in Cardiogenic Shock (ECLS-SHOCK).

[16] https://clinicaltrials.gov/ct2/show/NCT03813134 Testing the value of Novel Strategy and Its Cost Efficacy in Order to Improve the Poor Outcomes in Cardiogenic Shock (EUROSHOCK).

[17] J. Park, K. H. Choi, J. M. Lee et al., "Prognostic Implications of door-to-balloon time and onset-to-door time on mortality in patients with ST -segment-elevation myocardial infarction treated with primary percutaneous coronary intervention," Journal of American Heart Association, vol. 8, Article ID e012188, 2019.

[18] N. K. Kapur, M. A. Alkhouli, T. J. DeMartini et al., "Unloading the left ventricle before reperfusion in patients with anterior ST-segment-elevation myocardial infarction," Circulation, vol. 139, no. 3, pp. 337-346, 2019.

[19] M. B. Basir, T. L. Schreiber, C. L. Grines et al., "Effect of early initiation of mechanical circulatory support on survival in cardiogenic shock," The American Journal of Cardiology, vol. 119, no. 6, pp. 845-851, 2017.

[20] C. Delmas, E. Puymirat, G. Leurent et al., "Design and preliminary results of FRENSHOCK 2016: a prospective nationwide multicentre registry on cardiogenic shock," Archives of Cardiovascular Diseases, vol. 112, no. 5, pp. 343-353, 2019.

[21] A. Kimmoun, E. Novy, T. Auchet, N. Ducrocq, and B. Levy, "Hemodynamic consequences of severe lactic acidosis in shock states: from bench to bedside," Critical Care, vol. 19, no. 1, p. 175, 2015.

[22] J. C. Schefold, G. Filippatos, G. Hasenfuss, S. D. Anker, and S. von Haehling, "Heart failure and kidney dysfunction: epidemiology, mechanisms and management," Nature Reviews Nephrology, vol. 12, no. 10, pp. 610-623, 2016.

[23] R. R. Filho, L. L. Rocha, T. D. Corrêa, C. M. S. Pessoa, G. Colombo, and M. S. C. Assuncao, "Blood lactate levels cutoff and mortality prediction in sepsis-time for a reappraisal? a retrospective cohort study," Shock, vol. 46, no. 5, pp. $480-485,2016$.

[24] O. Kruse, N. Grunnet, and C. Barfod, "Blood lactate as a predictor for in-hospital mortality in patients admitted acutely to hospital: a systematic review," Scandinavian Journal of Trauma, Resuscitation and Emergency Medicine, vol. 19, no. 1, p. 74, 2011.

[25] Y. J. Park, D. H. Kim, S. C. Kim et al., "Serum lactate upon emergency department arrival as a predictor of 30 day inhospital mortality in an unselected population," PLoS One, vol. 13, no. 1, Article ID e0190519, 2018.

[26] M. Frydland, J. E. Møller, S. Wiberg et al., "Lactate is a Prognostic factor in patients admitted with suspected STelevation myocardial infarction," Shock, vol. 51, no. 3, pp. 321-327, 2019.

[27] J. Pöss, J. Köster, G. Fuernau et al., "Risk stratification for patients in cardiogenic shock after acute myocardial infarction," Journal of the American College of Cardiology, vol. 69, no. 15, pp. 1913-1920, 2017.

[28] K. A. Eagle, M. J. Lim, O. H. Dabbous et al., "A validated prediction model for all forms of acute coronary syndrome," JAMA, vol. 291, no. 22, pp. 2727-2733, 2004.

[29] E. W. Tang, C. K. Wong, and P. Herbison, "Global registry of acute coronary events (GRACE) hospital discharge risk score accurately predicts long-term mortality post acute coronary 
syndrome," American Heart Journal, vol. 153, no. 1, pp. 29-35, 2007.

[30] K. A. A. Fox, O. H. Dabbous, R. J. Goldberg et al., "Prediction of risk of death and myocardial infarction in the six months after presentation with acute coronary syndrome: prospective multinational observational study (GRACE)," BMJ, vol. 333, no. 7578, p. 1091, 2006.

[31] K. R. Bainey and P. W. Armstrong, "Clinical perspectives on reperfusion injury in acute myocardial infarction," American Heart Journal, vol. 167, no. 5, pp. 637-645, 2014. 